\title{
XLI. On the discharge of Electricity through exhausted tubes without electrodes
}

\section{J.J. Thomson M.A. F.R.S.}

To cite this article: J. J. Thomson M.A. F.R.S. (1891) XLI. On the discharge of Electricity through exhausted tubes without electrodes, Philosophical Magazine Series 5, 32:197, 321-336, DOI: $10.1080 / 14786449108620192$

To link to this article: http://dx.doi.org/10.1080/14786449108620192

曲 Published online: 08 May 2009.

Submit your article to this journal $\lceil\pi$

山 Article views: 12

Q View related articles $\square$

4 Citing articles: 27 View citing articles 5 
LONDON, EDINBURGH, AND DUBLIN

\title{
PHIIJOSOPHICAL MAGAZINE
}

\author{
AND \\ JOURNAL OF SCIENCE. \\ [FIFTH SERIES.] \\ $O C T O B E R 1891$.
}

XLI. On the Discharge of Electricity through Eirhausted Tubes without Electrodes. By J. J. Тномson, M.A., F.R.S., Cavendish Professor of Experimental Physies, Cambridge.*

THE following experiments, of which a short account was read before the Cambridge Philosophical Society last February, were originally undertaken to investigate the phenomena attending the discharge of Electricity through Gases when the conditions are simplified by confining the discharge throughout the whole of its course to the gas, instead of, as in ordinary discharge-tubes, making it pass from metallic or glass electrodes into the gas, and then out again from the gas into the electrodes.

In order to get a closed discharge of this kind we must produce a finite electromotive force round a closed circuit, and since we cannot do this by the forces arising from a distribution of electricity at rest, we must make use of the electromotive forces produced by induction. To break down the electric strength of the gas such forces must be very intense while they last, though they need not last for more than a short time. Forces satisfying these conditions occur in the neighbourhood of a wire through which a Leyden jar is discharged. During the short time during which the oscillations of the jar are maintained enormous currents pass through the wire, and as with a moderate-sized jar these currents change their direction millions of times in a second,

* Communicated by the Author.

Phil. Mag. S. 5. Vol. 32. No. 197. Oct. 1891. 
the electromotive force in the neighbourhood of the wire is exceedingly large. To make these forces available for producing an electrodeless discharge, all we have to do is to make the wire connecting the coatings of the jar the primary of an induction-coil of which the discharge-tube itself forms the secondary. The arrangements which I have employed for this purpose are represented in the accompanying diagram.

Fig. 1.
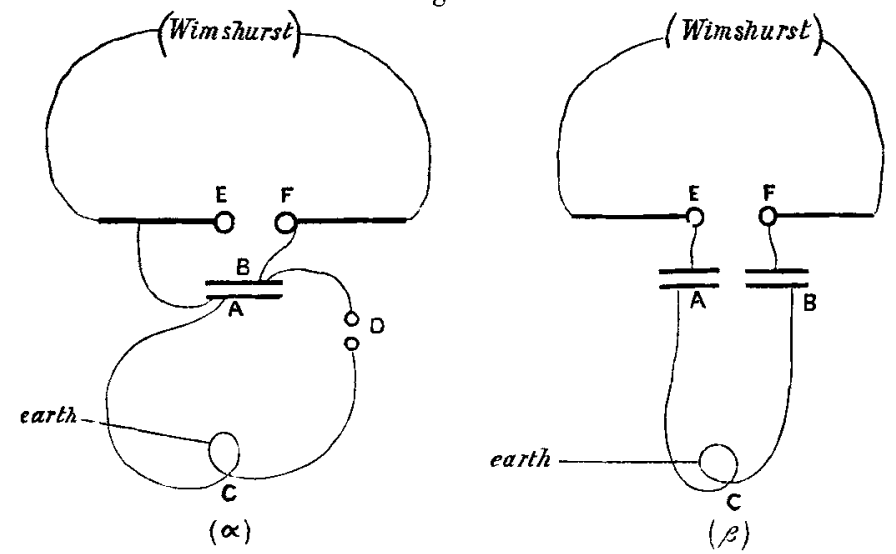

In ( $\alpha$ ) $\mathrm{A}$ is the inside coating of a Leyden jar : this is connected to $\mathrm{E}$, one of the poles of a Wimshurst electrical machine, or an induction-coil, the other pole $\mathrm{F}$ of the machine being connected to $B$ the outer coating of the jar. A C D is a wire connected to the inner coating of the jar, a few turns $C$ (which we shall call the primary coil) are made in this wire ; these turns are square if the discharge-tube is square, circular if the discharge-tube is a spherical bulb. The wire at $D$ is attached to an air-break, the other side of which is connected with the outer coating of the Leyden jar. The knobs of this air-break ought to be kept brightly polished. The loop $\mathrm{C}$ is connected to earth. The discharge-tubes, which were in general either rectangular tubes or spherical bulbs, were placed close to the turns of $C$. When the difference of potential between $A$ and $B$ is sufficiently large, a spark passes across the air-break, and the electrical oscillations set up produce a large electromotive force in the neighbourhood of the coil, sufficient under farourable circumstances to cause a bright discharge to pass through the vacuum-tubes. In some experiments the jars, at the suggestion of Prof. Oliver Lodge, were connected up differently, and are represented by $(\beta)$ in 
fig. 1. Two jars were used, the outside coatings of which, $A$ and $B$, were connected by the wire containing the primary coil $C$, the inside coating of the first jar was connected to one pole of the Wimshurst, that of the second to the other. With this method of arranging the jars no air-space is required, as the sparks pass between the terminals of the machine, and the polishing of these terminals is not nearly so important as that of the knobs of the air-break in the arrangement $(x)$.

Before proceeding to describe the appearance presented by the discharge, I will mention one or two points which may prove useful to any one who wishes to repeat the experiments. According to $\mathrm{my}$ experience the discharge is more easily obtained in bulbs than in square tubes, and with a Wimshurst machine than with an induction-coil. If an induction-coil is used a break which will transmit a large current ought to be substituted for the ordinary vibrating one supplied with such instruments. It is essential to success that the gas in the bulbs or tubes should be quite dry and at a suitable pressure; there is a pressure at which the brilliancy of the discharge is a maximum, and as in endeavouring to get at this pressure the exhaustion may be carried too far, it is convenient to use a form of mercury pump which will allow of the easy admission of a little gas; the pattern which I have used and found to answer very well is called the Lane-Fox pattern. When any gas is introduced it should be sent through sulphuric acid to get rid of any moisture that may be in it. Owing, I think, to the pressure in ordinary incandescent lamps being very different from that at which the discharge has its maximum brilliancy, I have met with very poor success in attempts to produce these discharges in already exhausted tubes such as incandescent lamps, though I have tried a considerable number by different makers; on the other hand, the radiometers which I hare tried allow the discharge to pass pretty readily, though it is interfered with by the vanes, and is not comparable in brilliancy with that obtained in home-made tubes and bulbs. I have obtained sparks easily with apparatus of the following dimensions : two gallon jars, the outside coatings connected by a wire about 2 yards long, the coil consisting of three or four turns, each about 3 inches in diameter. I have some bulbs which with this apparatus will give a bright discharge when the distance between the terminals of the Wimshurst is only $\frac{1}{4}$ inch ; these are, however, exceptionally good ; it more frequently takes a spark an inch or an inch and a half long to produce the discharge.

I find that Hittorf in Wiedemann's Annalen, xxi. p. 138, Z 2 
describes the light produced in a tube round which the wire connecting the coatings of a Leyden jar is twisted; the luminosity in Hittorf's experiments seems to have filled the tube, and not, as in the experiments described in this paper, been confined to a ring. It seems possible that the difference in the appearance in the tubes may have been due to the existence of an electrostatic action in Hittorf's experiments, the primary coil getting raised to a high potential before the discharge of the jar, and inducing a distribution of electricity over the inside of the glass of the tube ; on the passage of the spark the potential of the primary coil will fall, and the electricity on the glass redistribute itself; to effect this redistribution it may pass through the rarefied gas in the discharge-tube and produce luminosity.

In my experiments I took two precautions against this effect : in the first place I connected the primary coil to earth, so that its potential before discharge took place was unaltered, and as an additional precaution I separated the dischargetube from the primary by a cage made of blotting-paper moistened with dilute acid ; the wet blotting-paper is a sufficiently good conductor to screen off any purely electrostatic effects, but not a good enough one to interfere to an appreciable extent with the electromotive forces arising from rapidly alternating currents. In this way we can screen off any electrostatic effects due to causes which operate before the electrical oscillations in the jars begin; when once these have commenced, there ought not, I think, to be any separation of the electromotive forces into two parts, one being called electrostatic, the other electrodynamic. As this is a point on which it is desirable to avoid any misunderstanding, I hope to be excused if I treat it at some length.

In the mathematical treatment of the phenomena of the "Electromagnetic Field," it is customary and not inconvenient to regard the electromotive force as derived from two sources, or rather as consisting of two parts, one part being calculated by the ordinary rules of electrostatics from the distribution of electricity in the field, the other part being the differential coefficient of the vector potential with respect to the time. From a mathematical point of view, there is a good deal to be said for this division; the two forces have very distinct and sharply contrasted analytical properties. Thus the electrostatic force possesses the property that its line-integral taken round any closed curve vanishes, while the surfaceintegral of its normal component taken over a closed surface does not in general vanish. The "vector potential force," on the other hand, does not in general vanish when integrated 
round a closed curve, the surface-integral of its normal component taken over any closed surface, however, vanishes. When, however, our object is not so much mathematical calculation as the formation of a mental picture of the processes going on in the field, this division does not seem nearly so satisfactory, as the fundamental quantities concerned, the electrostatic and vector potentials, are both of considerable complexity from a physical point of view. We might judge that this diyision of the electromotive force into two parts, the one derivable from an electrostatic, the other from a vector, potential, is rather a mathematical device than a physical reality, from the fact which I pointed out in a report on Electrical Theories (B. A. Report, 1886), that though the electrostatic potential satisfies the mathematical condition of being propagated with an infinite velocity, the total electromotive force in the electromagnetic field travels with the velocity of light, and nothing physical is propagated at a greater velocity.

In an experimental investigation such as that described in this paper it is not so important that our method of regarding the phenomena should lead to the shortest analysis as that it should enable us to picture to ourselves the processes at work in the field, and to decide without much calculation how to arrange the experiments so as to bring any effect which may have been observed into greater prominence.

The method which I have adopted for this purpose is the one described by me in the Philosophical Magazine, March 1891 , and which consists in referring everything to the disposition and motion of the tubes of electrostatic induction in the field. These tubes are either endless, or have their ends on places where free electricity exists, every unit of positive electricity (the unit being the quantity of electricity on the atom of a univalent element) being connected by a unit tube to a unit of negative electricity, the tube starting from the positive electricity and ending on the negative. At any point in the field the electromotive intensity varies as the density of the tubes of electrostatic induction at that point. When the electricity and the tubes in the field are at rest, the tubes distribute themselves so that the electromotive intensity at any point is derivable from a potential function ; as soon, however, as the equilibrium is disturbed, the tubes move about and get displaced from their original positions, the disposition of tubes and therefore the electromotive intensity are changed, and the latter will no longer be derivable from a potential function, and according to the mathematical theory would be said to include forces due to electrostatic and electromagnetic induction. Acrording to our view. 
however, the cause of the electromotive intensity is the same in both cases, viz. the presence of tubes of electrostatic induction, and the electromotive intensity ceases to be derived from a potential, merely because the distribution of these tubes is not necessarily the same when they are moving about as when they are in equilibrium. It is shown, in the paper already referred to, that these tubes when in motion produce a magnetic force at right angles, both to their own direction and to that in which they are moving, the magnitude of the force being $4 \pi$ times the product of the strength of the tube, the velocity with which it is moving, and the sine of the angle between the direction of the tube and its direction of motion. In an electric field in which the matter is at rest, these tubes when in motion move at right angles to themselves with the velocity " $v$," that at which electrodynamic disturbances are propagated through the medium. $W$ e can easily show that, $K$ being the specific inductive capacity of the medium, the line-integral of $4 \pi / K$ times the density of these trubes taken round a closed circuit is equal to the rate of diminution of the number of lines of magnetic induction passing through the circuit. Thus, since the fundamental laws of electrodynamic action, viz. Faraday's law of induction and Ampère's law of magnetic force, follow from this conception of the field as produced by tubes of electrostatic induction moving at right angles to themselves with the velocity " $v$," and producing a magnetic force at right angles both to their own direction and to that in which they are moving, and proportional to the product of the strength of the tube and its velocity, it is a conception which will account for all the known phenomena of the field. It furnishes in fine a geometrical instead of an analytical theory of the field. It will also be seen that from this point of view the magnetic force, when introduced to calculate the electromotive forces arising from induction, logically comes in as an intellectual middle-man wasting mental effort.

We may thus regard the distinction between electrostatic and electromagnetic electromotive forces as one introduced for convenience of analysis rather than as having any physical reality. The only difference which I think could be made from a physical point of view would be to define those effects as electrostatic which are due to tubes of electrostatic induction having free ends, and to confine the term electromagnetic to the effects produced by closed endless tubes. It is only, however, when the electromotive forces are produced exclusively by the motion of magnets that all the tubes are closed; whenever batteries or condensers are used, open tubes are present in the field. 
It will be useful to consider here the disposition and motion of the tubes of electrostatic induction in the arrangement used to produce these electrodeless discharges. We shall take the case where two jars are used, as in $\beta$, fig. 1 , as being the more symmetrical.

Just before the discharge of the jar, the tubes of electrostatic induction will be arranged somewhat as follows:There will be some tubes stretching from one terminal of the electric machine to the other; others will go from the terminals to neighbouring conductors, the table on which the machine is placed, the floor and walls of the room, \&c. The great majority of the tubes will, however, be short tubes passing through the glass between the coatings of the jars. Let us now consider the behaviour of two of these tubes, one from the jar $A$, the other from $B$, when a spark passes between the terminals of the machine. Whilst the spark is passing these may be regarded as connected by a conductor; the tubes which originally stretched between them now contract, the repulsion they exerted on the surrounding tubes is destroyed so that these now crowd into the space between the terminals, the two short tubes under consideration now taking somewhat the form shown in fig. 2. These tubes being of opposite sign

Fig. 2.

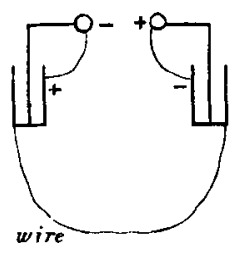

Fir. 4.

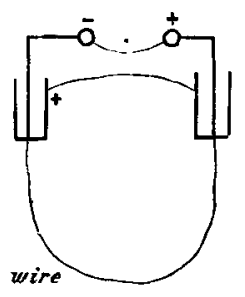

Fig. 3.

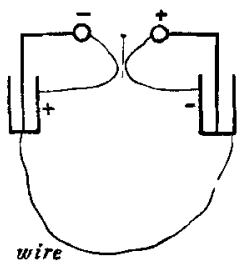

Fin. 5.

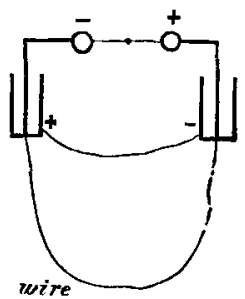

tend to run together; they do so until they meet as in fig. 3 , when the tubes break up as in fig. 4, the upper portion running into the spark-gap, where it contracts, while the lower 


\section{Prof. J. J. Thomson on the Discharge of Electricity}

portion rushes through the dielectric to discharge itself into the wire connecting the coatings of the jars, an intermediate position being shown in fig. 5 . These tubes while rushing through the dielectric produce, as already stated, magnetic forces; some of them on their way to the discharging wire will pass through the discharge-tube; if they congregate there in sufficient density, discharge will take place through the rarefied gas.

The discharge of the jar is oscillatory, and we have only followed the motion of the tubes during a part of the oscillation; when, however, this tube enters the wire between the jars a tube of opposite kind emerges from it; the same thing happens when the other portion enters the spark-gap. These go through the same processes as the tubes we have followed, but in the reverse order, until we get again two short tubes in the jars, but opposite in sign to the original ones; the process is then repeated and so on as long as the vibrations last.

In order to see what are the most advantageous dimensions to give to our apparatus, let us consider on what the maximum electromotive force in the secondary depends. Let us take the case of a condenser of capacity $\mathrm{C}$ discharging throngh a circuit whose coefficient of self-induction is L ; then, if the potential difference between the plates of the condenser is initially $V_{0}$, the current $\gamma$ at the time $t$ is (supposing as a very rough approximation that there is no decay in the vibrations) given by the equation

$$
\gamma=\frac{\mathrm{CV}_{0}}{\sqrt{\overline{\mathrm{LC}}}} \sin \frac{t}{\sqrt{\mathrm{LC}}}
$$

The rate of variation of this, $\dot{\gamma}$, is therefore

$$
\frac{\mathrm{V}_{0}}{\mathrm{~L}} \cos \frac{t}{\sqrt{\mathrm{LC}}} \text {. }
$$

So that if $\mathrm{M}$ is the coefficient of self-induction between the primary and a secondary circuit, the maximum electromotive force round the secondary is $M V_{0} / \mathrm{L}$, which for a given sparklength is independent of the capacity of the condenser. In practice it is advisable, however, to have as much energy in the jars to start with as possible, and better results are got with large jars than with small ones. Using a six-plate Wimshurst machine I got very good results with two "gallon jars;" with a large induction-coil the best results were got with two "pint and a half jars."

The best number of turns to use in the primary coil $\mathrm{C}$ 
depends upon the size of the leads; if all the circuit were available for this coil one turn would give the largest electromotive force, because though for a given rate of change of the current in the primary the effect on the secondary increases with the number of turns, the rate of change of the current varies inversely as the self-induction of the primary, so that if all the circuit is in the coil $\mathrm{C}$, since an increase in the number of turns will increase the self-induction of the circuit faster than the mutual induction, it will diminish the electromotive force round the secondary. In practice, however, it is not possible to have the whole of the wire connecting the coatings of the jar in the coil $\mathrm{C}$; and in this case an increase in the number of turns may increase the mutual induction more than the selfinduction, and so be advantageous. The best result will be obtained when the self-induction in the coil $\mathrm{C}$ is equal to that of the remainder of the circuit. It is very easy to find by actual trial whether the addition of an extra turn of wire is beneficial or the reverse. The brightness of the discharge depends upon the time of the electrical oscillations as well as upon the magnitude of the electromotive force. Thus, in an experiment to be described later, the brilliancy of the discharge was increased by putting self-induction in the leads which, though it diminished the intensity of the electromotive force, increased the time-constant of the system. When the discharge-tube was square and the coil $\mathrm{C}$ had also to be square, it was found most convenient to make it of glass tubing bent into the required form and filled with mercury. When, however, the discharge was required in a bulb, the primary coil was made of thick gatta-percha-covered copper wire wound round a beaker just large enough to receive the exhausted bulb. There is sometimes considerable difficulty in getting the first discharge to pass through the bulb, though when it has once been started other discharges follow with much less difficulty. The same effect occurs with ordinary sparks. It seems to be due to the splitting up of the molecules by the first discharge; some of the atoms are left uncombined and so ready to conduct the discharge, or else when they recombine they form compounds of smaller electric strength than the original gas. When the discharge was loath to start, I found the most effectual way of inducing it to do so was to pull the terminals of the Wimshurst far apart and then, after the jars had got fully charged, to push the terminals suddenly together. In this way a long spark is obtained, which, if the pressure of the gas is such that any discharge is possible, with the means at our disposal will generally start the discharge. 


\section{Appearance of the Discharge.}

Let us suppose that we have either a square tube placed outside a square primary or a bulb placed inside a circular coil of wire, and that we gradually exhaust the dischargetube, the jars sparking all the time. At first nothing at all is to be seen in the secondary, but when the exhaustion has proceeded until the pressure has fallen to a millimetre or thereabouts, a thin thread of reddish light is seen to go round the tube situated near to but not touching the side of the tube turned towards the primary. As the exhaustion proceeds still further, the brightness of this thread rapidly increases, as well as its thickness; it also changes its colour, losing its red tinge and becoming white. On continuing the exhaustion the luminosity attains a maximum, and the discharge passes as an exceedingly bright and well-defined ring. On continuing the exhaustion, the luminosity begins to diminish until, when an exceedingly good vacuum is reached, no discharge at all passes. The pressure at which the luminosity is a maximum is very much less than that at which the electric strength of the gas is a minimum in a tube provided with electrodes and comparable in size to the bulb. The pressure at which the discharge stops is exceedingly low, and it requires long continued pumping to reach this stage. We see from these results that the difficulty which is experienced in getting the discharge to pass through an ordinary vacuum-tube when the pressure is very low is not altogether due to the difficulty of getting the electricity from the electrodes into the gas, but that it also occurs in tubes without electrodes, though in this case the critical pressure is very much lower than when there are electrodes. In other words, we see that as the state of the bulb approaches that of a perfect vacuum its insulating power becomes stronger and stronger. This result is confirmed by several other experiments of a different kind which will be described later.

The discharge presents a perfectly continuous appearance, with no sign of striation, of which I have never observed any trace on any of these discharges, though I must have observed many thousands of them under widely different conditions.

\section{Action of a Magnet on the Discharge.}

The discharges which take place in these tubes and bulbs are produced by periodic currents, so that the discharges themselves are periodic, and the luminosity is produced by currents passing in opposite directions. As this is the case, 
it seemed possible that the uniformity of the luminosity seen in the discharge was due to the superposition of two stratified discharges in opposite directions, the places of maximum luminosity in the one fitting into those of minimum Iuminosity in the other. Since these discharges are in opposite directions, they will be pushed opposite ways when a magnetic force acts at right angles to them, the discharges in opposite directions can thus be separated by the application of a magnetic force and examined separately. In the experiment which was tried with this object, a square tube was used placed outside the primary, the tube at one or two places being blown out into a bulb so as to allow of the wider separation of the constituent discharges. When one of these bulbs was placed in a magnetic field where the force was at right angles to the discharge, the luminous discharge through the bulb was divided into two portions which were driven to opposite sides of the bulb; each of these portions was of uniform luminosity and exhibited no trace of striation. It was noticed, however, in making this experiment that the discharge seemed to have much greater difficulty in pessing through the tube when the electromagnet was on than when it was off. This observation was followed up by several other experiments, and it was found that the discharge is retarded in a most remarkable way by a magnetic force acting at right angles to the line of discharge. This effect is most strikingly shown when the discharge passes as a ring through a spherical bulb. If such a bulb is placed near a strong electromagnet, it is easy to adjust the length of spark so that when the magnet is off a brilliant discharge passes through the bulb, while when the magnet is on no discharge at all can be detected. The action is very striking, and the explanation of it which seems to fit in best with the phenomena I have observed is that the discharge through the rarefied gas does not rise to its full intensity suddenly, but as it were feels its way. The gas first breaks down along the line whore the electromotive intensity is a maximum, and a small discharge takes place along this line. This discharge produces a supply of dissociated molecules along which subsequent discharges can pass with greater ease. Thus under the action of these electric forces the gas is in a state of unstable equilibrium, since as soon as any small discharge passes through it the gas becomes electrically weaker and less able to resist subsequent discharges. When the gas is in a magnetic field, the magnetic force acting on the discharge produces a mechanical force which displaces the molecules taking part in the discharge from the line of maximum electric intensity, and thus subsequent discharges 
do not find it any easier to pass along this line in consequence of the passage of the previous one. There will not, therefore, be the same instability in this case as in the one where no magnetic force acted upon the gas. A confirmation of this view is, I think, afforded by the appearance presented by the discharge when the intensity of the magnetic field is reduced, so that the discharge just, but only just, passes when the magnetic field is on. In this case the discharge, instead of passing as a steady fixed ring, flickers about the tube in a very undecided way.

If the strength of the magnetic field is reduced still further, so that the discharge passes with some ease, the bright ring which, when no magnetic force is acting, is in one plane, is changed into a luminous band situated between two planes which intersect along a diameter of the bulb at right angles to the magnetic force. These planes are inclined at a considerable angle, one being above and the other below the plane of the undisturbed ring. This displacement of the ring by the magnetic force shows that it consists of currents circulating tangentially round the ring.

This action of a magnet on a discharge flowing at right angles to its lines of force is not, however, the only remarkable effect produced by a magnet on the discharge. When the lines of magnetic force are along the line of discharge, the action of the magnet is to facilitate the discharge and not to retard it as in the former case. The first indication of this was observed when the jars were connected, as in (a) in fig. 1. The earth-connexion being removed, in this case there is a glow from the glass into the bulb, due to the redistribution of the electricity induced on the glass by the primary when it is at a high potential before the spark passes. If the primary is connected to earth by a circuit with an air-break in it, the intensity of the glow may be altered at will by adjusting the length of the air-break; when the air-space is very small there is no glow; when it is long the glow is bright. The bulb in which the discharge was to take place was placed on a piece of ebonite over the pole of an electromagnet, and the air-space in the earth-connexion of the primary was adjusted so that when the magnet was off no glow was observed in the tube. When the magnet was on, however, a glow radiating in the direction of the lines of magnetic force was produced, which lasted as long as the magnet was on, and died away rapidly, but not instantaneously, when the magnet was taken off. In this case the discharge seems to be much easier along the lines of magnetic force.

The following experiment shows that this effect is not 
confined to the glow-discharge, but is also operative when the discharge passes entirely through the gas. A square tube $\mathrm{ABCD}$ (fig. 6) is placed outside the primary EFGH, the lower part of the discharge-tube CD being situated between the poles L M of an electromagnet. By altering the length of spark of the Wimshurst machine, the electromotive intensity

Fig. 6.

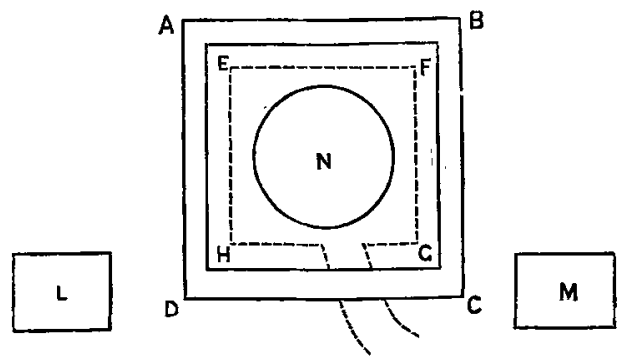

acting on the secondary can be so adjusted that no discharge passes round the tube $A B C D$ when the magnet is off, whilst a bright discharge occurs as long as the magnet is on. The two effects of the magnet on the discharge, viz. the stoppage of the discharge across the lines of magnetic force, and its acceleration along them, may be prettily illustrated by placing in this experiment an exhausted bulb $\mathrm{N}$ inside the primary; then the spark-length can be adjusted so that when the magnet is off the discharge passes in the bulb, and not in the square tube, while when the magnet is on the discharge passes in the square tube, and not in the bulb.

The experiments on the effect of the magnetic field on the discharge were tried with air, carbonic acid, and oxygen, but I could not detect any difference in the behaviour of the gases.

The explanation of the longitudinal effect of magnetic force seems more obscure than that of the transverse effect; it is possible, however, that both may be due to the same cause, for if the feeble discharge which we suppose precedes the main discharge branches away at all from the line of main discharge, the action of the magnetic force when it is along the discharge will tend to bring these branches into the main line of discharge ; and thus there will be a greater supply of dissociated molecules along the main line of discharge, and therefore an easier path for the subsequent discharges when the magnetic force is acting than when it is absent.

It is perhaps not necessary to assume that the mechanical action of the magnetic force is on a small discharge preceding 


\section{4}

Prof. J. J. Thomson on the Discharge of Electricity

the main one; the action of the magnetic force on the chain of polarized molecules which are formed before the discharge passes might produce an effect equivalent to that which we have supposed was produced on an actual discharge.

The chain of polarized molecules would be affected in the following way:- The magnetic field due to the electromagnet consists of tubes of electrostatic induction moving about: these tubes, as well as the direction in which they are moving, are at right angles to the lines of magnetic force. The short tubes of electrostatic induction which join the atoms in the molecules of the gas will, under the influence of the electric forces, set themselves parallel to the direction of the electromotive intensity at each point.

Thus, when the magnetic force is at right angles to the line of discharge, tubes of electrostatic induction parallel to those in the molecules will be moving about in the field; and since parallel tubes exert attraction and repulsion on each other, the molecular tubes will be knocked about and their efforts to form closed chains made much more difficult by the action of the magnet. On the other hand, when, the lines of magnetic force are parallel to the discharge, the moving tubes are at right angles to those in the molecules, and will not disturb them in the attempt to form chains along the line of magnetic force, they will in fact assist them in doing so by preventing all attempts in directions across the lines of force.

Prof. G. F. Fitzgerald has suggested to me in conversation that this action of a magnet on the discharge might be the cause of the "streamers" which are observed in the aurora; the rare air being electrically weaker along the lines of magnetic force than at right angles to them will cause the discharge in the direction of those lines to be the brightest.

\section{Discharge through different Gases.}

I have examined the discharge through air, carbonic acid, hydrogen, oxygen, coal-gas, and acetylene. As I bave already mentioned, at the highest pressures at which the discharge passes through air, the discharge is reddish, and gets brighter and whiter at lower pressures. If the discharge is examined through a spectroscope, the lines in the spectrum coincide with those obtained by sparking through air in the ordinary way with a jar in the circuit. The relative brightness of the lines in the spectrum of the discharge without electrodes varies very much with the pressure of the gas and the length of spark in the jar circuit. With a long spark in this circuit, and the pressure such as to give a bright white 
discharge, the spectrum is very much like that of the ordinary jar-discharge in air. When, however, the pressure is so low that the discharge passes with difficulty, a few lines in the spectrum shine out very brightly, whilst others become faint, so faint indeed sometimes that if the air-spectrum were not thrown into the field of view of the spectroscope at the same time, they might pass unnoticed. Three lines which are very persistent, the first a citron-green, the second a more refrangible green, and the third a blue, I am inclined to think must be due to mercury vapour from the pump.

I am indebted to Prof. Liveing for the loan of a very fine direct-vision spectroscope, and to him and Mr. Robinson, of the Cambridge Chemical Laboratory, for valuable advice in the attempts which I made to photograph the spectra of some phosphorescent glows mentioned below.

I should like to call attention to the advantages for spectroscopic purposes which attend this method of producing the discharge ; it is easily done either by an ordinary electrical machine or an induction-coil. An intensely bright discharge is got, and there is no danger of complication arising from the spectrum of the gas getting mixed with that of the electrodes.

\section{Discharge in Oxygen.}

By far the most remarkable appearance is presented when the discharge passes through oxygen, for in this gas the bright discharge is succeeded by a phosphorescent glow which lasts for a considerable time; indeed, with a strong discharge it may remain visible for more than a minute. When the discharges succeed one another pretty rapidly, the phosphorescence is so strong that it hides the successive bright discharges, and the tube seems permanently full of a bright yellow fog. We can thus by the use of this gas convert the intermittent light given by the bright discharge into a continuous one.

Perhaps the most striking way of showing this phosphorescence is to use a long tube, about a metre long and 6 or 7 centimetres in diameter, with a bulb blown in the middle, the primary coil being twisted round this bulb. Then, when the sparks pass between the jars, a bright ring-discharge passes through the bulb, from which, as if shot out from the ring, the phosphorescent glow travels in both directions along the tube, moving slowly enough for its motion to be followed by the eye. It cannot, therefore, be produced by the direct action of the light from the spark on the gas in the tube, for 
if it were, the glow would travel with the velocity of light. It is necessary to mention this point, for the light from these discharges has great powers of producing phosphorescence.

The glow seems to consist of gas which has been in the path of the discharge, and whose molecules have been split up by it and projected from the line of discharge. This gas which, when projected, is in a peculiar state, by a process of chemical combination gradually returns to its original condition, and it is while it is in this state of transition from its new condition to the old that it phosphoresces. If this is the case we should expect that the period of phosphorescence would be shortened by raising the temperature. On trying the experiment I found that this took place to a very marked extent. A discharge-bulb filled with oxygen at a low pressure was placed over a Bunsen burner ; before the bulb got hot each bright discharge was succeeded by a bright afterglow, but as the bulb got hotter and hotter the glow became fainter and fainter, and at last ceased to be visible, though the bright ring was still produced at each discharge of the jar. When the Bunsen was taken away and the bulb allowed to cool, the glow reappeared.

The spectrum of the afterglow is a continuous spectrum, in which I could not detect the superposition of any bright lines. The only gas besides oxygen in which I have been able to detect any afterglow is air, though in this case the range of pressure within which it is exhibited is exceedingly small ; indeed it is often by no means an easy matter to get a bulb filled with air into the state in which it shows the glow. The spectrum of the air-glow showed bright lines ; I thought myself that I could see a very faint continuous spectrum as well. Some friends, however, who were kind enough to examine the spectrum, though they could see the bright lines clearly enough, were of opinion that there was nothing else visible. I endeavoured to photograph it, but without success, so that the existence of a continuous spectrum for this glow must be considered doubtful.

When the discharge passes through acetylene, the first two or three discharges are a bright apple-green, the subsequent ones, however, are white, and as the green discharge does not reappear, we must conclude that the acetylene is decomposed by the discharge.

[To be continued.] 